\title{
The Relationship Between Immediate Response Syndrome and the Expectations Toward Artificial Intelligence and Robots in Taiwan
}

\author{
Hiroko Kanoh \\ Yamagata University, Yamagata, Japan
}

\author{
Dienfang Chou \\ Tzu Chi University, Hualien County, Taiwan
}

\begin{abstract}
With the popularity of smart phones, the style of interpersonal communication and habits has changed in Taiwan. Some of the Social Networking Service (SNS) users have interpersonal anxiety and suspicion. The purpose of this paper is to analysis the immediate response syndrome for college students in Taiwan. Moreover, Artificial Intelligence (AI) and humanoid robots are become more widely applied used and accepted in the future. The relationship between "Immediate Response Syndrome" and the expectations toward artificial intelligence and robots were also clarified. For determining the Immediate Response Syndrome in Taiwan, this paper followed the checking list proposed by Kanoh. Online Google Forms were used to design surveys and forms for the students in Hualien, Taiwan. From April 2016 to October 2016, 235 samples were collected. Research findings showed that Taiwanese college students spend too much time online. Although the time of internet usage is not related to self-evaluation, but according to correlation analysis, it is positively related to Immediate Response Syndrome. Moreover, Immediate Response Syndrome is not related to self-evaluation, but is positively related to the attitudes toward artificial intelligence and robots. People who are more qualified for Immediate Response Syndrome have higher expectations toward artificial intelligence and robots.
\end{abstract}

Keywords: Immediate Response Syndrome, Artificial Intelligence (AI), robots, Social Networking Service (SNS)

\section{Introduction}

In recent years, as the increased of the internet usage, the communication between people has become more convenient. With the popularity of mobile phones and well developed communication applications, the life styles of people have been shaped changed dramatically.

According to the non-governmental organization "Institute for Information Industry" (2017/05/01), in Taiwan, the average person has four accounts for community websites. Facebook (90.9\%) and LINE (87.1\%) are the first and second (60.4\%) most popular, followed by PTT (37.8\%), Instagram (32.7\%), WeChat, Twitter, and Dcard, etc.

Institute for Information Industry (2017/04/14) also shows found that more than 90 percent of Taiwanese people are using smart phones. On average, each person has 3.6 digital devices. More than 70 percent of them check their smart phones while watching TV, and more than 50 percent of them chat with friends using social 
networking apps or check on the websites via smart phones or tablet devices while watching TV.

With the popularity of smart phones, the style of interpersonal communication and habits has changed. For example, the "READ" function provided by social networking apps may improve the immediacy and efficiency of interpersonal communication, but may also increase anxiety.

Dailyview (2014) — an internet big data analysis website—points out that the most anxious situation for Social Networking Service (SNS) users is when they saw see the "READ" on the application, but haven't received any feedbacks.

104 IT Corporation (2014) found that about $30 \%$ of office workers were in a bad mood when their messages were ignored. The United Daily News (2014) also reported this problem as a psychiatric sign concern - LINE Syndrome-because people didn't receive any reply. Some of the SNS users have interpersonal anxiety and suspicion.

Therefore, to maintain friendship, it has become necessary for young people to respond to messages from their friends as soon as possible. However, with the increasing number of users of SNS, it is not easy to maintain relationships via SNS. Checking every friend's daily post or replying to every message immediately takes time and energy. Immediate Response Syndrome is a term that describes a condition of mental instability caused by an obsession with the anxiety need to respond to messages immediately. Kanoh (2016a) pointed out the Immediate Response Syndrome is a pathological feature in the SNS culture.

Over the past few years, Google, Microsoft, IBM, Facebook, Apple, Amazon, Yahoo, and other famous technology companies, have launched a number of artificial intelligence inventions, including-Google translation, personal voice assistant, Google's unmanned vehicles, Unmanned Aerial Vehicle, human-computer robots, systems for diagnosis of cancer, and automatic stock automatic trading-These are the examples of applications of artificial intelligence. Artificial intelligence brings convenience, and efficiency, and can be applied in all areas. Surely, this is the trend for the future. The rise of artificial intelligence will impact the labor market, and will take over many functions currently performed by humans.

Robots have mainly been used in the manufacture manufacturing industry, and service robots can only be seen in movies. After But after Foxconn launched Softbank's Pepper humanoid robots in Taiwan in 2016 (Kao, 2016), the robots are likely to become more widely applied used and accepted in the future.

Due to the trend, Kanoh (2016b) proposed a hypothesis that people who have a high level of interest in new information devices, such as smart phones, and usage them frequently, toward new information devices, such as Mart phones, may be associated with have higher expectations toward for artificial intelligence and robots. Therefore, the purpose of this paper is to clarify the relationship between "Immediate Response Syndrome" and the expectations toward artificial intelligence and robots.

\section{Method}

The subject of this paper is to clarify the relationship between Immediate Response Syndrome and the expectations toward artificial intelligence and robots in Taiwan. In order to understand the Immediate Response Syndrome in Taiwan, this paper will follow the checking list below proposed by Kanoh (2016b):

(1) Care about mobile phone cannot be apart without them;

(2) Always uses the mobile phone;

(3) Will feel anxious when the phone is not within the reach of the range;

(4) Will experience irritable when the phone is not within the reach of the range; 
(5) Will feel nervous when the phone is not within the reach of the range;

(6) Had received expensive phone bills;

(7) Feels restless if did not receive a reply after waiting for more than 15 minutes;

(8) Replies immediately even received a message at midnight;

(9) Replies immediately even received a message while taking shower;

(10) Replies immediately even received a message while eating;

(11) Has almost nearly lost important relationships due to the mobile phone; and

(12) Can't live without mobile phone.

The checking standard of the normal range is from 0 to 4 , and while 5 to 8 can be considered having indicates a tendency toward Immediate Response Syndrome, from 9 to 12 can be defined as Immediate Response Syndrome. Moreover, the expectations toward artificial intelligence and robots will use the chosen items based on the research findings of Kanoh (2015).

In addition, Kanoh (2015) has compiled 10 predictions for humanoid robots in Japan. They are (1) Emergency Response; (2) Disaster Relief; (3) Working for Dangerous and Difficult Jobs; (4) Manage child care and housework; (5) Serving Customers; (6) Providing nursing care; (7) As a Farm laborer; (8) As a porter; (9) Working for service industry; and (10) As a family member. These 10 predictions can be seen as the significance of the humanoid robots.

When someone sees the "READ" mark on the social networking apps, but didn't get any response. This sometimes would cause different levels of anxiety. Usually, people with such kind of syndrome have lower self-esteem and higher social anxiety (Jia, Luo, \& Lin, 2014). In the interpersonal interactions, they are with higher levels of defensive cognitive and emotional reactions, if things do not go with their expectations. For example, if they do not receive any respond response, they will feel out of control, unsafe, and anxious. Therefore, in order to explore the relationship between Immediate Response Syndrome and the expectations toward artificial intelligence and robots in Taiwan, this paper also examines the self-esteem by scale as below:

(1) I am content with myself;

(2) I feel like a loser;

(3) I consider myself a person of good qualities;

(4) I can do most of the things that people can do;

(5) I have nothing to be proud of;

(6) I feel like I'm useless;

(7) I feel I have the same value as others;

(8) I wish I could show more respect for myself;

(9) I tend to think of myself as a loser; and

(10) I take a positive attitude towards myself.

\section{Results}

The data used in this paper was collected from April 2016 to October 2016 in Hualien County, Taiwan. Online Google Forms were used to design surveys and forms for the students in Tzu Chi University and Tzu Chi University of Science and Technology. Of the 235 samples were collected within six months, $71.9 \%$ were females and $28.1 \%$ males. $74.5 \%$ of samples were from Tzu Chi University, and $24.3 \%$ are from Tzu Chi University of Science and Technology, and the rest remaining 3\% were from other universities or non-college 
students. $96.6 \%$ of the respondents aged between 18 to $22.99 .1 \%$ of them were using smart phones, $7.2 \%$ were using general mobile phones, $29.8 \%$ have tablet devices, and $77.9 \%$ have computers. The data shows the average time of daily internet usage is 342.99 minutes, meaning that university students on averagely spend 5.7 hours a day, almost 40 hours a week online. Obviously, compared to other countries, Taiwanese college students spend more hours online. For example, in the UK young people aged between 16 and 24 spend more than 27 hours a week (Anderson, 2015).

According to Kanoh (2015), the checking standards, described above, for Immediate Response Syndrome, from 0 to 4 is the normal range, and from 5 to 8 is considered having Immediate Response Syndrome tendency, from 9 to 12 was identified as Immediate Response Syndrome. Through this standard, there are $57.9 \%$ of respondents are in the normal range, $36.6 \%$ of the samples have Immediate Response Syndrome tendency, and $6 \%$ can be seen as having Immediate Response Syndrome.

Nevertheless, according to correlation analysis, the daily time spent using the internet is positively related to Immediate Response Syndrome. In other words, the more time people spend on the net, they will be to more likely respond to messages immediately. In addition, the time of internet usage is also positively related to mobile phone separation anxiety. The heavy internet users feel nervous, anxious, and irritable when their mobile phone is not within the reach of the range. They care greatly about mobile phones and cannot leave home without them.

Table 1

Relationship Between Immediate Response Syndrome and Daily Time Online

\begin{tabular}{lll}
\hline & & The number of Immediate Response Syndrome \\
\hline \multirow{2}{*}{ The number of Immediate Response } & Pearson correlation & $0.254^{* *}$ \\
Syndrome & Sig. (2-tailed) & 0.000 \\
& $\mathrm{~N}$ & 229 \\
\hline
\end{tabular}

Note. ${ }^{* *}$ Correlation is significant at the 0.01 level (2-tailed).

Furthermore, the result showed there were $51.5 \%$ of male and $60.4 \%$ of female don't have Immediate Response Syndrome and this tendency, compared with the finding in Japan, the percentage were $76 \%$ of male and $72 \%$ of female (Kanoh, 2016a), thus, It is determine the Immediate Response Syndrome in Taiwan is more serious than in Japan.

Besides, the expectation toward artificial intelligence and robots, Emergency Response (90.6\%), Disaster Relief (86.4\%) and Dangerous and Difficult Jobs (81.3\%) were the top three most expected, and Manage child care and house work (20.4\%), Serving Customers (23.8\%) and as a Family Member (26.4\%) were most unexpected. Thus, the college students in Taiwan expect robots active as rescuer or hard working machine, especially in some dangerous and dirty scenarios, not as partners to live with together.

Moreover, self-evaluation is positively related to the attitudes toward artificial intelligence and robots. People who have positive self-evaluation are looking forward to living with robots, are interested in them, and regard robots them as helpful company. Instead of the contrast, people who have negative self-evaluation are not interested in robots and even loathe them.

However, as other previous studies showed the negative impact of using the instant message application is not significant than we imagine(Huang \& Chen, 2015), the result in this survey shows the time of internet usage is not related to gender and self-evaluation. It is not correlated with the attitudes and expectations toward 
artificial intelligence and robots, but positively related to the living area and environment. The data shows that people who live in the mountains, fishing, and agricultural areas spend much longer more time on the internet than people who live in the urban cities.

Table 2

Immediate Response Syndrome With Gender

\begin{tabular}{|c|c|c|c|c|c|}
\hline & & & \multicolumn{2}{|c|}{ Gender } & \multirow{2}{*}{-Total } \\
\hline & & & MALE & FEMALE & \\
\hline \multirow{6}{*}{ Immediate Response Syndrome } & \multirow{2}{*}{ NO } & Count & 34 & 102 & 136 \\
\hline & & $\%$ within Gender & $51.5 \%$ & $60.4 \%$ & $57.9 \%$ \\
\hline & \multirow{2}{*}{ Tendency } & Count & 26 & 59 & 85 \\
\hline & & $\%$ within Gender & $39.4 \%$ & $34.9 \%$ & $36.2 \%$ \\
\hline & \multirow{2}{*}{ YES } & Count & 6 & 8 & 14 \\
\hline & & $\%$ within Gender & $9.1 \%$ & $4.7 \%$ & $6.0 \%$ \\
\hline \multirow{2}{*}{ Total } & & Count & 66 & 169 & 235 \\
\hline & & $\%$ within Gender & $100.0 \%$ & $100.0 \%$ & $100.0 \%$ \\
\hline
\end{tabular}

In addition, the Immediate Response Syndrome is related to gender: Males are more qualified for likely to show this Syndrome. Immediate Response Syndrome is also related to living area and environment, in the same way that these factors are related to which is the same as the relationship between the hours of internet usage and living area and environment. People who live in the mountains, fishing, and agricultural areas are more qualified for susceptible to Immediate Response Syndrome.

Besides, the Immediate Response Syndrome is not related to self-evaluation, but is positively related to the attitudes toward artificial intelligence and robots. People who are more qualified for Immediate Response Syndrome have higher expectations toward artificial intelligence and robots.

Overall, research findings showed that Taiwanese college students spend too much time online. Although the time of internet usage is not related to self-evaluation, but according to correlation analysis, it is positively related to Immediate Response Syndrome. As stated by Kanoh (2016a), Immediate Response Syndromes were classified into "unconscious type" and "needed type". In this paper, didn't investigate this issue thoroughly. Therefore, as a suggestion, future research may focus on the classification of usage types in Taiwan.

In conclusion, although with the popularity of mobile phones and well developed communication applications, the reaction toward "READ" on the application are gradually concerned than before. But the Immediate Response Syndrome also worth to be discuss, especially the online hours and Immediate Response Syndrome were serious among the young people in Taiwan. Moreover, the research finding shows people who have negative self-evaluation are not interested in robots and even loathe them. Perhaps create an image of Artificial Intelligence (AI) and robots as a partner in daily life via mobile phones or other devices may increase the acceptance, especially artificial intelligence and robots have become the trend for the future,

\section{References}

Anderson, E. (2015). Teenagers spend 27 hours a week online: how internet use has ballooned in the last decade. Telegraph Media Group. $\quad$ Retrieved from http://www.telegraph.co.uk/finance/newsbysector/mediatechnologyandtelecoms/digital-media/11597743/Teenagers-spend-2 7-hours-a-week-online-how-internet-use-has-ballooned-in-the-last-decade.html

Dailyview. (2014). Top 10 anxious situations of using internet. Retrieved from http://dailyview.tw/Daily/2014/07/01

Huang, Y., \& Chen, S. (2015). A research on instant message application usage. Graphic Communication Arts Journal, 346: 345-352. 
Institute for Information Industry. (2017/04/14). 3 years 3 times! More than 70 percent of them check their smart phones while watching TV. Retrieved from http://www.iii.org.tw/Press/NewsDtl.aspx?nsp_sqno=1923\&fm_sqno=14

Institute for Information Industry. (2017/05/01). More than 80 percent of Taiwanese people enjoy using Facebook and Line, the average person has four accounts for community websites. Retrieved from http://www.iii.org.tw/Press/NewsDtl.aspx?nsp_sqno=1934\&fm_sqno=14

Jia, W. L., Luo, D., \& Lin, Y. T. (2014).Why you didn't reply the message: Discussing the interpersonal problems of internet users by attachment theory. Counseling \& Guidance, 347: 42-46.

Kanoh, H. (2015). Occurrence of LINE caste: Why friendship is easily destroyed by KIDOKU MUSHI (Read Ignoring). The 40th Annual Conference of Japanese Society for Information and Systems in Education (pp. 341-342). Japanese Society for Information and Systems in Education.

Kanoh, H. (2016a). Analysis of the immediate response syndrome for university students. Technical Report of Information Processing Society of Japan, HCI-167, 3:1-7. Information Processing Society of Japan.

Kanoh, H. (2016b). The relationship between Immediate Response Syndrome and the expectations toward artificial intelligence and robots. The 34th Annual Conference of the robotics society of Japan, RSJ2016AC3Y3-04. The robotics society of Japan.

Kao, Y. (2016). Salary is under 22K Robots are on duty. Zencosmos, 138: 26-33.

The United Daily News. (2014). Didn't receive any reply: LINE Syndrome. Retrieved from https://health.udn.com/health/story/5964/368266

104 IT Corporation. (2014). Use tablet or mobile phone for 2 hours every day, the evolution of workplace communication. Retrieved from https://www.104.com.tw/area/media/article/detail/id/167121573302869731/categiry/3 